\title{
Novos Dispositivos para Oclusão de Defeitos Cardíacos Congênitos
}

\author{
Áurea J. Chaves
}

$\mathbf{N}$ esta edição da Revista Brasileira de Cardiologia Invasiva, artigos originais e editoriais trazem muitas novidades, relacionadas, em especial, às novas opções para a oclusão de defeitos cardíacos congênitos.

Pedra et al., do Hospital do Coração (São Paulo, SP) e do Instituto Dante Pazzanese de Cardiologia (São Paulo, SP), relatam sua experiência inicial com a prótese Figulla para o fechamento percutâneo da comunicação interatrial tipo ostium secundum. Trata-se de prótese semelhante à Amplatzer, mas com diferenças que trazem vantagens e desvantagens, detalhadamente discutidas no artigo. Essa contribuição condensa a vivência de um grupo com vasta experiência na oclusão percutânea de defeitos septais, com o uso de diversas próteses e em variados cenários anatômicos. Zabal, do Instituto Nacional de Cardiologia Ignácio Chávez (México, DF, México), em seu editorial, analisa a corrida tecnológica que levou ao desenvolvimento de diversos dispositivos de oclusão, que se adaptam aos variados tipos, formas e tamanhos de defeitos e que hoje podem tratar cerca de $80 \%$ de todos os defeitos do septo atrial.

Esteves et al., do Instituto Dante Pazzanese de Cardiologia (São Paulo, SP), apresentam a primeira experiência brasileira com a prótese PREMERE, especialmente desenvolvida para a correção do forame oval patente. Pacientes com eventos cerebrais embólicos prévios, evidenciados por tomografia computadorizada ou ressonância nuclear magnética, e diagnóstico de forame oval patente foram tratados com sucesso, comprovado por ecocardiogramas transesofágicos seriados com injeção de microbolhas. Uhlemann, do Olgahospital (Stuttgart, Alemanha), em editorial correspondente, comenta as controvérsias ligadas às duas técnicas que competem como método de escolha no diagnóstico de forame oval patente: o ecocardiograma transesofágico e o Doppler transcraniano, ambos com injeção de microbolhas e manobra de Valsalva. Lembra a variabilidade morfológica do forame oval patente, as indicações de seu tratamento baseadas nas diretrizes, e a necessidade de se comparar os resultados dos novos dispositivos com o risco natural de eventos neurológicos dessa afecção.

Outra área explorada nesta edição é relacionada à intervenção coronária percutânea no extremo da faixa etária. Thomas Júnior et al., do Hospital Bandeirantes (São Paulo, SP), mostram os resultados da intervenção coronária percutânea primária em pacientes com infarto agudo do miocárdio com mais de 80 anos. Pimentel Filho et al., do Hospital Beneficência Portuguesa de São Paulo (São Paulo, SP) e do Hospital Evangélico Samaritano de Campinas (Campinas, SP), vão mais além e expõem os resultados da intervenção coronária percutânea em nonagenários, segmento da população que triplicou nas últimas décadas. Esses autores trazem em seu artigo o interessante índice de Katz, que avalia a competência funcional do paciente em seis funções da vida diária. Gebara, do Instituto do Coração (InCor) do Hospital das Clínicas da Faculdade de Medicina da Universidade de São Paulo (São Paulo, SP) e do Hospital TotalCor (São Paulo, SP), e Wajngarten, do InCor (São Paulo, SP), em editorial que comenta esses manuscritos, nos alertam a respeito da necessidade de individualizar a indicação de revascularização miocárdica nos muito idosos, frequentemente excluídos dos estudos clínicos controlados, da disponibilidade dos demais índices, que ajudam a tornar mais objetivas as indicações terapêuticas, e, sobretudo, da necessidade de haver o diálogo médico-paciente para se ter em perspectiva as expectativas e preferências desses pacientes muito especiais.

Outro artigo, muito original, de Cardoso et al., do Instituto de Cardiologia do Rio Grande do Sul (Porto Alegre, RS) e da Universidade Federal do Rio Grande do Sul (Porto Alegre, RS), nos insere no desconhecido mundo da gestão dos laboratórios de hemodinâmica. O planejamento orçamentário, ferramenta que permite calcular e estimar custos, melhora a aplicação dos recursos financeiros disponíveis, sem prejudicar a qualidade dos serviços médicos oferecidos. Castello, do Hospital Bandeirantes (São Paulo, SP), intervencionista com MBA Executivo em Saúde pela Fundação Getúlio Vargas e verdadeiro expert no assunto, lembra a complexidade do planejamento orçamentário, que deve ser individualizado para que possa ser aplicado às distintas realidades dos diferentes serviços. Ressalta ainda a necessidade dos laboratórios de hemodinâmica em oferecer os melhores serviços com menores custos, responsabilidade social e metas arrojadas de crescimento. 
Em nossa primeira edição de 2010, reestruturamos o Corpo Editorial, que passará a contar com três editores associados e quatro coeditores:

- Editores associados: Alexandre Abizaid (Instituto Dante Pazzanese de Cardiologia - São Paulo, SP), Carlos Augusto Cardoso Pedra (Instituto Dante Pazzanese de Cardiologia - São Paulo, SP) e Rogério Sarmento-Leite (Instituto de Cardiologia - Porto Alegre, RS).

- Coeditores: Carlos A. H. de Magalhães Campos (Instituto do Coração - São Paulo, SP e Hospital Ibiapaba/CEBAMS - Barbacena, MG), Cristiano de Oliveira
Cardoso (Instituto de Cardiologia - Porto Alegre, RS), Daniel Chamié (INTERCAT - Rio de Janeiro, RJ) e J. Ribamar Costa Jr. (Instituto Dante Pazzanese de Cardiologia - São Paulo, SP).

Ampliamos, também, o Conselho Editorial, que passará a contar com Marinella P. Centemero (Instituto Dante Pazzanese de Cardiologia - São Paulo, SP) e Renato D. Lopes (Duke Clinical Research Institute Durham, Estados Unidos).

Nossas boas-vindas a todos!

Áurea J. Chaves

Editora 